\title{
Les innovations dans le secteur ferroviaire : un système d'innovation en transition entre régime à impulsion étatique et régime marchand
}

\author{
Innovations in the railway sector: an innovation system in transition \\ between state impulsion regime and market oriented regime
}

\author{
Nicolas Raimbault ${ }^{1}$, Corinne Banquart ${ }^{2}$, Philippe Poinsot $^{3}$ \\ ${ }^{1}$ IRT Railenium, France, et Department of Urban Development and Mobility, Luxembourg Institute of Socio-Economic \\ Research (LISER), Esch-sur-Alzette, Luxembourg, nicolas.raimbault@liser.lu \\ ${ }^{2}$ IFSTTAR/SPLOTT, Université Paris-Est, Marne-La-Vallée, France, corinne.blanquart@ifsttar.fr \\ ${ }^{3}$ Laboratoire Ville Mobilité Transport (LVMT, UMR-T 9403), Ecole des Ponts, IFSTTAR, UPEM, UPE, Marne-La-Vallée, \\ France, philippe.poinsot@enpc.fr, philippe.poinsot@u-pem.fr
}

RÉSUMÉ. L'innovation est aujourd'hui considérée comme indispensable au secteur ferroviaire pour résoudre les difficultés que celui-ci connaît. Afin d'analyser les processus d'innovation dans ce secteur, nous adoptons une vision large de la production dans le ferroviaire, l'envisageant dans sa globalité et sa complexité. Cette approche montre que le secteur ferroviaire français se situe, sous l'effet de diverses mutations, et particulièrement de la montée en puissance des règles européennes de concurrence, dans un entre-deux, entre " impulsion étatique » et " régime marchand " (Boyer, 2015). Cette transition engendre des contradictions et limite la capacité à innover du secteur. En se fondant sur la notion de "système sectoriel d'innovation et de production » (Malerba, 2004) et en s'appuyant sur une trentaine d'entretiens semi-directifs auprès d'acteurs du secteur, cet article identifie des " nœuds ", des points de tension, dans les processus d'innovation et dans lesquels les contradictions du système se cristallisent, et définit les contours d'un agenda pour des recherches futures.

ABSTRACT. Innovation is now considered as being essential for the railway sector to solve the difficulties it faces. In order to analyze the innovation processes in this sector, we adopt an overall view of production in the rail system by considering it as a whole and with its complexity. This approach demonstrates that the French railway sector, due to diverse transformations, and particularly because of the rise in European competition rules, is currently in limbo between "state impulsion" and "market oriented regime" (Boyer, 2015). This transition entails to contradictions and limits the capacity to innovate the sector. Based on the notion of the "sectoral system of innovation and production" (Malerba, 2004) and about 30 in depth interviews of actors of the sector, this article identifies several "nodes", points of tension, in the innovation processes and in which the contradictions of the system concentrate. In this way, the article proposes the outline of an agenda for future research.

MOTS-CLÉS. secteur ferroviaire, processus d'innovation, système sectoriel d'innovations et de production, coordination d'acteurs, régime à impulsion étatique, régime marchand.

KEYWORDS. railway sector, innovation process, sectoral system of innovations and production, coordination of actors, state impulsion regime, market oriented regime.

\section{Introduction}

Le monde ferroviaire a longtemps été un monde de l'ingénierie et de la technologie. De la conception à l'exploitation, de la réalisation d'infrastructures à la fabrication du matériel roulant, sa technicité en fait un système de transports naturellement proche des progrès technologiques et de l'innovation. Toutefois, en France, depuis le TGV, aucune innovation majeure n'a véritablement marqué la filière, et le ferroviaire est aujourd'hui concurrencé par le transport aérien low-cost, les autres formes de mobilités partagées ou encore plus récemment par les autocars longue-distance. Des ruptures technologiques majeures influencent par exemple l'automobile, débouchant notamment sur le véhicule autonome. En matière de transport, l'innovation semble dès lors en dehors du ferroviaire. 
L'innovation est aujourd'hui considérée comme indispensable au secteur ferroviaire pour résoudre les difficultés que celui-ci connaît. D'une part, on assiste à un accroissement de la concurrence intermodale qui se traduit par la stagnation, voire le recul, des parts de marché du rail (en particulier pour le fret en France) (Ministère de l'Environnement, de l'Energie et de la Mer, 2016). D'autre part, les industriels et les opérateurs du secteur font face à une concurrence de plus en plus forte des entreprises chinoises et indiennes (Ingénieurs et Scientifiques de France, 2014 ; SETRA/CERTU, 2013). Des budgets conséquents sont ainsi consacrés à soutenir l'innovation. Par exemple, l'initiative européenne commune Shift2Rail (S2R) ${ }^{1}$ consacre 920 millions d'euros sur six ans pour soutenir l'innovation en matière ferroviaire, et notamment l'innovation ouverte, collaborative, entre les acteurs du secteur.

Alors que la littérature académique se focalise principalement sur la question de la libéralisation du ferroviaire (encadré 1) pour résoudre ses problèmes (voir par exemple Crozet, 2001; Nash, 2008, 2010), il parait essentiel, pour comprendre les processus d'innovation dans ce secteur et ses ressorts, d'avoir une vision plus large de la production dans le ferroviaire afin de l'envisager dans sa globalité et sa complexité. Aujourd'hui, c'est l'ensemble du secteur qui est bouleversé par un certain nombre d'évolutions économiques et sociétales et de réformes d'ordre institutionnel. Ces aspects déterminants pour l'innovation sont très peu abordés par la littérature académique alors qu'il s'agit d'un enjeu identifié par les historiens du ferroviaire, notamment dans le contexte d'européanisation du système ferroviaire (Fourniau, 2003). Ces mutations impliquent des problèmes dans les modes de coordination et d'interaction entre acteurs. Or, comme le souligne la littérature sur les «systèmes d'innovation » (SI), loin d'être un processus purement individuel ou entrepreneurial, l'innovation est plutôt le résultat des interactions entre différents acteurs insérés dans des systèmes institutionnels et sociaux (Amable, 2003). En ce sens, nous nous inscrivons dans la perspective de recherche dessinée par K. Speck analysant le TGV en tant que SI (2003), en l'élargissant au secteur ferroviaire tout entier.

Le processus de libéralisation du secteur ferroviaire dans l'Union Européenne se met en place à la suite de la directive $n^{\circ} 1991 / 440$ (29 juillet 1991) qui recommande une distinction claire entre les missions de gestionnaire d'infrastructure et de services de transport. Il se matérialise en France par la loi du 13 février 1997 avec la séparation du gestionnaire d'infrastructure, Réseau Ferré de France (RFF), et l'exploitant historiques (SNCF).

Entre 2001 et 2007, trois paquets ferroviaires sont proposés par la Commission européenne et votés par le Parlement européen et le Conseil européen avec pour objectif d'établir un espace unique européen en matière de transport. Chaque paquet porte sur un ou plusieurs des quatre volets suivants : l'ouverture à la concurrence, la sécurité, l'interopérabilité et le développement du corridor RTE-T.

Le premier paquet (adopté le 26 mars 2001) s'inscrit dans le prolongement direct de la directive $n^{\circ} 1991 / 440$ et vise l'ouverture à la concurrence du fret international. Le deuxième paquet (29 avril 2004) conduit à la création de l'agence ferroviaire européenne (ERA) et renforce les volets sécurité et interopérabilité du réseau à grande vitesse. Elle vise aussi à rendre effective la libéralisation du fret international au $1^{\mathrm{er}}$ janvier 2006 et du fret européen au $1^{\mathrm{er}}$ janvier 2007. En France, le transport international de fret est ouvert à la concurrence en 2003 et le fret européen en 2006. De plus, la loi du 5 janvier 2006 acte la création de l'Etablissement Public de Sécurité Ferroviaire (EPSF) en charge de la sécurité et du développement des transports en France. Le troisième paquet ( 23 octobre 2007) consiste d'une part, à une clarification des règles relatives aux Obligations de Services

\footnotetext{
${ }^{1}$ S2R a été créée en 2014 à l'initiative de l'association européenne de l'industrie ferroviaire (UNIFE) dans le cadre du programme de recherche européen H2020 : http://shift2rail.org/
} 
Publics (règlement OSP) et d'autre part, à la mise en place de l'ouverture à la concurrence des services de transport international de voyageurs au plus tard en 2010.

La loi du 9 décembre 2009 crée en France la Direction de la Circulation Ferroviaire (DCF), entité indépendante de la $\mathrm{SNCF}$, en charge des circulations ferroviaires, et la transposition du troisième paquet ferroviaire conduit à la création de l'Autorité de Régulation des Activités Ferroviaires (ARAF) dont la mission est de "garantir la non-discrimination dans l'accès au réseau » (Crozet, 2014, p. 21). Le $1^{\text {er }}$ janvier 2010, la création de la branche Gares \& Connexions (G\&C) vise un égal traitement des entreprises ferroviaires dans leur accès aux gares.

Le 21 novembre 2012, le Parlement européen et le Conseil européen adoptent une directive (directive 2012/34/UE) qui confirme les libéralisations déjà prévues et précise que la séparation comptable entre gestionnaire d'infrastructure et exploitant ferroviaire, au sein de la même entreprise, est jugée conforme aux directives européennes. Le $1^{\text {er }}$ janvier 2015 entre en vigueur en France une loi qui conduit à la création, le $1^{\mathrm{er}}$ juillet 2015 , d'un nouveau groupe public ferroviaire SNCF. Celuici assure le pilotage de deux établissements publics : SNCF Réseau qui est issu de la fusion de RFF, SCNF Infra et de la DCF, c'est-à-dire de l'ensemble des services en charge de la gestion du réseau ferroviaire national; SNCF Mobilités qui est l'exploitant.

Le quatrième paquet ferroviaire a été proposé par la Commission européenne en janvier 2013 et devrait être la dernière étape du processus de libéralisation. Celui-ci vise plus précisément l'ouverture des Lignes à Grande Vitesse (LGV) en «open access », qui seront effectives au plus tard en décembre 2020, et l'ouverture des services conventionnés (TER et Trains d'équilibre du territoire) par des procédures d'appel d'offre. Ces derniers seront possibles à partir de 2019 puis obligatoires en 2023.

Pour plus de détails : Crozet, 2014.

Encadré 1. Le processus de libéralisation du secteur ferroviaire en Europe et sa déclinaison en France

Le secteur ferroviaire français est ainsi, sous l'effet de ces diverses mutations, dans un entre-deux qui engendre des contradictions dans le système et limite sa capacité à innover. En effet, celui-ci se trouve dans une période de transition d'un régime que Boyer qualifie de régime à «impulsion étatique », c'est-à-dire une configuration dans laquelle « le public [est] au coeur de l'innovation [...] », vers un « régime marchand» où le marché est le « vecteur essentiel de l'innovation » (Boyer, 2015, p. 142). L'ambition de cet article n'est pas de donner une vision complète et exhaustive des processus d'innovation dans le ferroviaire, mais plus modestement de définir les contours d'un agenda pour des recherches futures visant à mieux les comprendre dans ce secteur particulier qu'est le ferroviaire. Plus précisément, il cherche à identifier des «nœuds», des points de tension, dans les processus d'innovation et dans lesquels les contradictions du système se cristallisent pour le cas français.

Pour ce faire, nous mobilisons d'une part, la grille d'analyse des « systèmes sectoriels d'innovation et de production » (SSIP ; Malerba, 2004). En effet, le recours aux SSIP nous parait être une bonne clef de lecture des mutations et des contradictions du ferroviaire, à même de tenir compte du caractère hybride du ferroviaire, à la fois public et privé, et de ses caractéristiques particulières comme industrie de réseau : rôle important des infrastructures ayant une longue durée de vie et étant très dépendantes de la puissance publique ; complexité des systèmes d'acteurs publics et privés ; enfin, des mécanismes de marché fortement influencés par la réglementation et les financements publics (Theys, 2005 ; Pérennes, 2014). D'autre part, nous nous appuyons sur une trentaine d'entretiens semi-directifs, réalisés dans le 
cadre du projet de recherche Manifer ${ }^{2}$, auprès de différents acteurs du secteur: constructeurs de matériel roulant, opérateurs de transport, institutions représentatives, autorités de régulation, centres de ressources, bureaux d'ingénierie, gestionnaires et constructeurs d'infrastructures, chargeurs et ministère des transports (tab. 1). Dans ces entretiens, les acteurs interrogés expliquent de manière développée à la fois comment les innovations qu'ils jugent marquantes ont émergé et se sont diffusées mais aussi les freins et leviers propres aujourd'hui dans le secteur ferroviaire.

\begin{tabular}{|c|c|}
\hline Type d'acteur & Nombre d'entretiens \\
\hline constructeurs de matériel roulant & 8 \\
\hline opérateurs de transport & 4 \\
\hline institutions représentatives & 3 \\
\hline autorités de régulation et ministère & 3 \\
\hline centres de ressources & 3 \\
\hline bureaux d'ingénierie et de conseil & 3 \\
\hline gestionnaires d'infrastructures & 2 \\
\hline constructeurs d'infrastructures & 1 \\
\hline chargeurs & 4 \\
\hline Instituts de recherche et universités & \\
\hline
\end{tabular}

Tableau 1. Entretiens réalisés par type d'acteur

Après avoir précisé, dans la partie 1, les mutations que le secteur ferroviaire a connues ces dernières années, nous mettrons l'accent, dans la partie 2, sur un certain nombre de tensions, les nœuds, qui émergent de ces mutations et constituent des leviers d'innovation privilégiés. Ce travail aboutira ainsi à une meilleure compréhension des contradictions que connaît actuellement le système ferroviaire français et européen.

\section{Le ferroviaire comme "système sectoriel d'innovation et de production »}

La notion de système d'innovation n'est pas directement opérationnelle et laisse entière la question des éléments à prendre en compte (acteurs, institutions, interactions, organisations, interfaces) et de l'échelle (nationale, sectorielle). Dans cet article, nous privilégions une entrée sectorielle, celle du « système sectoriel d'innovation et de production » (SSIP) (Malerba, 2004) qui nous semble à même de rendre compte des mutations du ferroviaire. Le recours au SSIP a aussi pour objectif de bâtir une grille d'analyse visant à distinguer les différents mécanismes pesant sur les principaux nœuds des processus d'innovation ferroviaire qui seront abordés dans la section 2 (fig. 1). Un SSIP est défini comme « [a] system (group) of firms active in developing and making a sector's products and in generating and utilizing a sector's technologies » (Breschi et Malerba, 1997, p. 131). Plus précisément, il repose sur trois éléments clefs : le régime technologique et d'apprentissage (Breschi et al, 2000) (1.1), les acteurs et leurs réseaux ensuite (1.2) et enfin les institutions (1.3) (Malerba, 2002).

\footnotetext{
${ }^{2}$ Ce projet a été financé par l'ANR et l'IRT Railenium. Il s'est déroulé de janvier 2015 à septembre 2016 et avait pour objectif d'identifier et d'analyser les besoins et les leviers de performance du système d'innovation ferroviaire. Nous tenons à remercier Marjorie André qui a réalisé la majorité des entretiens sur lesquels nous nous appuyons dans cet article.
} 


\subsection{Le régime technologique et d'apprentissage : entre inertie et émergence de nouveaux paradigmes}

Le régime technologique et d'apprentissage, articulant une base de connaissances et des processus d'apprentissage, influence les sources et l'organisation sectorielles de l'innovation. En effet, la plus ou moins grande facilité de diffusion, d'appropriation ou au contraire l'idiosyncrasie d'une base de connaissances détermine les possibilités d'arrivée de nouveaux innovateurs ou, au contraire, la stabilité des acteurs déjà en place (possibilité pour les innovateurs de maintenir leur avantage) (Malerba, 2002).

Dans le ferroviaire, cette notion renvoie aux spécificités techniques du ferroviaire et à ce que les acteurs nomment la « culture ferroviaire ». D'une part, le ferroviaire est considéré comme une industrie en réseau dont les possibilités et la dynamique d'innovation sont intimement liées à l'infrastructure et à ses contraintes en termes de coût, de durée de vie, d'inertie. D'autre part, le régime technologique et d'apprentissage correspond aussi à l'existence de référentiels cognitifs partagés par la majorité des acteurs du ferroviaire, la « culture ferroviaire », également marquée par l'inertie et la valorisation de l'expérience. Par exemple, le «retour d'expérience» est considéré comme le principal et l'incontournable mode de validation d'un projet (voir 2.2.1). D'après les acteurs interrogés, cette base de connaissances serait peu favorable à toute innovation radicale, notamment en termes de règles, d'organisation et d'ingénierie. La culture ferroviaire conduirait davantage à des innovations incrémentales concernant la fiabilité, la maintenance et l'optimisation de l'existant.

Mais, en parallèle, et même s'il est un facteur historique de fermeture du secteur vis-à-vis de nouveaux entrants en raison des grandes spécificités du ferroviaire, le régime technologique ferroviaire semble aujourd'hui connaître de réelles mutations découlant de l'importation de nouvelles compétences depuis l'extérieur du secteur, notamment celles de la transition numérique. Les modalités de formation et d'apprentissage des agents du ferroviaire apparaissent comme l'un des facteurs explicatifs à la fois de cette prégnance de la «culture ferroviaire » et de l'émergence de ces nouveaux paradigmes (voir section 2.2).

\subsection{Les réseaux d'acteurs: vers la fin du leadership de l'opérateur}

Comme l'indique Malerba (2002), un SSIP se caractérise et se différencie des autres secteurs par le rôle des acteurs interagissant au sein de réseaux. Les acteurs pertinents correspondent aux entreprises du secteur, aux fournisseurs, utilisateurs ou usagers des produits et aux organisations non marchandes, gouvernementales et universitaires notamment. Les réseaux sont définis comme les interactions marchandes et non marchandes dans lesquels les agents sont engagés.

L'étude des réseaux d'acteurs met en évidence une évolution considérable au sein du secteur ferroviaire. En effet, le ferroviaire constitue une filière historiquement structurée à l'échelle nationale par les opérateurs, longtemps en monopole (par exemple, la SNCF en France et la Deutsche Bahn en Allemagne), qui nourrissaient des relations privilégiées avec un constructeur national (Alstom en France, Siemens en Allemagne). Par exemple, pour la France, la SNCF exerçait un fort leadership dans la conception du matériel roulant et de l'infrastructure, et donc dans nombre d'innovations passées dans ces domaines ${ }^{3}$.

Les règles de concurrence au cœur de l'européanisation des institutions, non seulement dans les services de transport mais plus largement dans toute l'industrie (dont l'industrie ferroviaire), et en matière de marchés publics, ainsi que le principe de séparation des activités d'exploitation ferroviaire et de gestion de l'infrastructure (encadré 1), ont remis en cause ce leadership et conduit au désengagement des opérateurs de l'organisation de la filière (Speck, 2003). La mise en place d'appels d'offres internationaux systématiques pour les commandes de matériel ferroviaire, c'est-à-dire la montée en puissance d'un régime marchand au sein du secteur ferroviaire, entre par exemple en

\footnotetext{
${ }^{3}$ L'exemple emblématique de cette période est bien sûr le TGV (Speck, 2003).
} 
contradiction avec l'intervention des opérateurs en amont dans la conception du matériel, et donc avec leur participation directe à l'innovation en la matière. De même, la séparation entre exploitants et gestionnaires d'infrastructure ont conduit, d'un côté, les opérateurs à s'affirmer de plus en plus nettement comme des entreprises de services de mobilités (telle SNCF mobilités) et de l'autre côté, les gestionnaires comme des entreprises de services d'infrastructure (telle SNCF réseau) (Ingénieurs et Scientifiques de France, 2014). Charge maintenant aux constructeurs de matériels ferroviaires, qui s'affirment comme des ensembliers, et à leurs fournisseurs de répondre à ces besoins.

Parallèlement, en lien avec l'élargissement de la base de connaissances, des réseaux intersectoriels émergent, à même de catalyser des innovations en matière d'applications numériques, de nouveaux matériaux ou encore de nouvelles organisations.

\subsection{Les institutions : règles sectorielles, organismes de soutien à l'innovation et gouvernance des services et infrastructures de transport}

Les institutions regroupent ici d'une part l'ensemble des normes, habitudes, routines, pratiques, standards, règles et lois dans lesquels les acteurs du secteur évoluent et d'autre part les organismes de représentation et d'animation du secteur (Malerba, 2002). Nous ajoutons à cette liste les politiques de transport ou plus exactement les modalités de la gouvernance des infrastructures et des services de transport (nationaux et locaux), c'est-à-dire la coordination des acteurs publics et privés impliqués dans ces différentes actions publiques (Le Galès, 1995). En effet, nous avons indiqué, dès l'introduction, l'importance de l'action publique, souvent locale (régionale/urbaine), comme constituant une spécificité du secteur ferroviaire, secteur hybride public et privé. La gouvernance des transports ferroviaires peut, dès lors, constituer un frein aussi bien qu'un levier de l'innovation.

Trois grandes mutations nous semblent particulièrement importantes à souligner concernant les institutions: le développement d'un corpus règlementaire et normatif européen cadrant les coordinations marchandes, l'émergence de nouveaux organismes de financement de l'innovation et d'importantes évolutions dans la gouvernance des transports.

Premièrement, dans la mesure où la montée en puissance du régime marchand remet en cause nombre de relations informelles entre les acteurs structurants du secteur, les règles établies aux échelles européenne et nationale, les normes, définies par les opérateurs et industriels du secteur et, plus largement, le processus d'appel d'offre par les opérateurs, sont de fait devenus des outils cruciaux de coordination entre acteurs. En effet, elles garantissent non seulement le respect des critères de sécurité mais aussi l'interopérabilité des matériels et l'ouverture des marchés, et donc une concurrence industrielle, sur les différents réseaux européens. Ces institutions restent très spécifiques au ferroviaire et sont caractérisées par une forte inertie, leur révision supposant un consensus sectoriel (Fontanel et Christeller, 2016). Ce fonctionnement semble donc laisser peu de place aux approches innovantes (voir 2.1.2). De plus, la gestion de la propriété intellectuelle devient également plus cruciale, en tant qu'incitation à l'innovation (Crampes et Encaoua, 2003), le nombre d'acteurs prenant part à l'innovation devenant plus important et les logiques concurrentielles s'étant accrues entre eux.

Deuxièmement, ont récemment émergé plusieurs organismes de soutien à l'innovation comme l'IRT Railenium, des pôles de compétitivité (Moveo, Nov@log et surtout I-Trans) à partir des années 2000, ou encore S2R à l'échelle européenne. Ces derniers renforcent les ressources proposées par les institutions de recherche plus anciennes (programme de recherche PREDIT du ministère des transports, l'IFSTTAR qui succède à l'INRETS en tant qu'institut public de recherche sur les transports). Si ces organismes apparaissent comme des déterminants de divers projets d'innovation dans le matériel roulant, les infrastructures et l'ingénierie notamment, les acteurs interrogés sont plutôt critiques à l'endroit de ces dispositifs. En effet, selon ces derniers, ces organismes de soutien ne répondraient qu'imparfaitement à leurs besoins pour des raisons d'inadéquation institutionnelle ou territoriale. 
Enfin, troisièmement, deux évolutions dans la gouvernance des transports semblent avoir joué un rôle important d'après les acteurs interrogés : la décentralisation et l'arrivée de nouveaux entrants. La décentralisation de certains services de transport a conduit à rapprocher les décideurs (politiques, administratifs) et les utilisateurs des transports ferroviaires (Crozet et Desmaris, 2011). En effet, la décentralisation confie aux autorités organisatrices des transports (AOT, c'est-à-dire aux collectivités locales en charge des transports urbains ou régionaux) la maîtrise d'ouvrage sur les commandes de matériels et d'équipements ferroviaires concernant les services dont elles ont la charge (Ingénieurs et Scientifiques de France, 2014). Ces dernières peuvent impulser des innovations sensibles aux demandes des usagers. La seconde évolution dans la gouvernance des transports est le choix de confier la construction et la gestion de certaines infrastructures à des concessionnaires conduisant à l'arrivée de nouveaux entrants porteurs de pratiques et d'approches différentes de ces métiers, et en ce sens pouvant être des leviers d'innovation.

Ainsi, le SSIP ferroviaire a connu des mutations importantes ces dernières années sous l'effet, d'une part, des évolutions du système productif national et international, telles que l'approfondissement de la concurrence internationale, l'augmentation des prix de l'énergie et la transition numérique, et, d'autre part, des transformations de l'Etat et de l'action publique, en particulier la montée en puissance de l'Union européenne et des règles de concurrence, la décentralisation et les nouveaux modes d'organisation et de financement de la recherche (fig. 1). Celles-ci conduisent aujourd'hui à un certain nombre de contradictions dans le SSIP ferroviaire qui sont particulièrement visibles dans ce que nous qualifions de "nœuds », c'est-à-dire des moments particuliers dans la production ferroviaire ou l'organisation de la filière, où à la fois ses contradictions se cristallisent mais constituent aussi des enjeux et des terrains privilégiés de futures innovations dans le ferroviaire. Il s'agit maintenant de préciser ces nœuds.

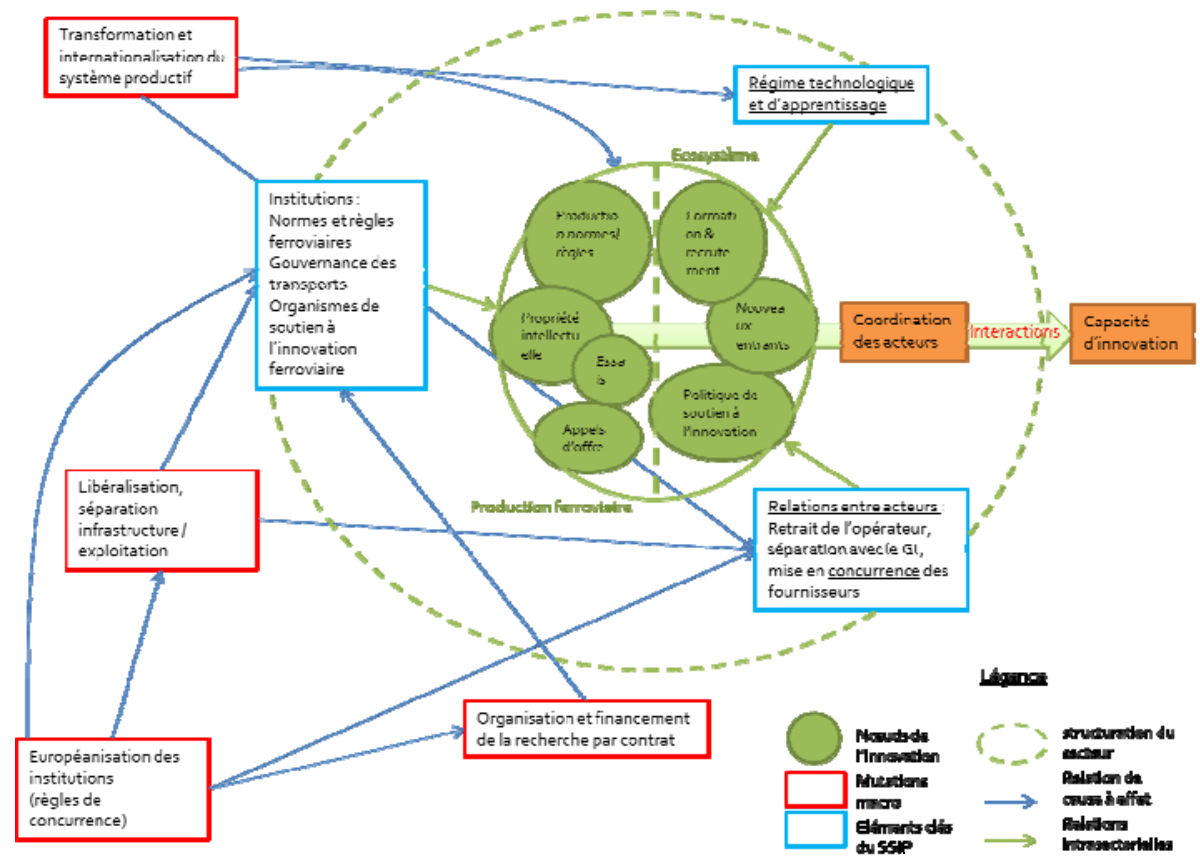

Figure 1. Le système ferroviaire d'innovation et de production et ses nœuds

\section{Les nœuds du SSIP ferroviaire : modes de coordination des acteurs du ferroviaire en régime marchand}

Les mutations du SSIP ferroviaire cristallisent un certain nombre de nœuds dans le processus d'innovation du ferroviaire. Ils renvoient d'une part à la coordination des acteurs aux différentes phases de la production ferroviaire (2.1) et d'autre part aux bouleversements de l'écosystème ferroviaire (2.2). Il s'agit maintenant de clarifier et de préciser chacun de ces nœuds afin de mettre en évidence plusieurs leviers d'innovation pour le secteur ferroviaire. 


\subsection{La coordination des acteurs aux différentes phases de la production ferroviaire}

Une partie des nœuds mis en évidence correspond aux relations entre acteurs qui se tissent à différents moments de la production du matériel ferroviaire : lors des appels d'offres des exploitants (2.1.1), en amont autour de la production des normes et des règles ferroviaires, qui déterminent notamment les exigences de sécurité, et du processus d'autorisation (2.1.2), enfin en matière d'essais (2.1.3) et de gestion de la propriété intellectuelle (2.1.4).

\subsubsection{Les appels d'offres et les cahiers de charge comme outils de coordination}

Les appels d'offres constituent l'un des principaux moments de coordination et d'organisation de la concurrence au sein du secteur. Le principe de mise en concurrence systématique et à l'échelle internationale des fournisseurs découle des règles européennes. Comme nous l'avons souligné dans la section 1, cela a profondément remis en cause l'implication historique des opérateurs en matière d'innovations dans les matériels ferroviaires. Celle-ci passait par l'établissement de relations privilégiées avec certains constructeurs, pratique qui structurait les relations entre acteurs de la filière. Les opérateurs se sont donc non seulement retirés de la conception mais de plus n'échangent plus avec leurs fournisseurs.

Cependant, le principal outil de coordination entre exploitants et constructeurs est resté le même : le cahier des charges envoyé à toutes les entreprises candidatant au marché. Le cahier des charges permettait historiquement aux exploitants de définir précisément les matériels qu'ils commandaient aux constructeurs. Les exploitants concentraient les compétences en ingénierie et les constructeurs, formant alors un tissu industriel relativement éclaté, se comportaient peu ou prou en sous-traitants (Machefert-Tassin, 2003).

Or, dans le cadre de la mise en concurrence des constructeurs, l'outil des cahiers des charges est conservé. Son recours est même renforcé : ils sont appréhendés comme la condition d'une mise en concurrence transparente et équitable. C'est pourquoi, au moins dans le cas de la SNCF, les spécifications dans les appels d'ordre, auxquelles doivent répondre les fournisseurs pour être retenus, sont restées généralement très importantes. Dans ces conditions, les constructeurs peuvent difficilement négocier les critères de ces cahiers des charges. Ainsi, un tel outil permet-il d'organiser une concurrence sur les prix pour construire le matériel principalement pensé par l'opérateur mais rend très difficile une différenciation des constructeurs par l'innovation.

Ce système associant mise en concurrence et cahiers des charges très spécifiques met finalement les constructeurs face à une injonction contradictoire. D'un côté, ils sont enjoints à proposer des produits innovants, cette tâche n'étant plus du ressort des exploitants. De l'autre, ils doivent répondre à un cahier des charges techniquement précis, les empêchant d'être réellement force de proposition et les soumettant essentiellement à un arbitrage sur les coûts. De plus, ce système débouche sur une organisation de la production industrielle à la commande, c'est-à-dire enclenchée uniquement en réponse aux appels d'offres des opérateurs, ce qui réduit les séries et donc limite les possibilités d'économies d'échelle (Ribeill, 2003).

Cependant, émergent de nouvelles approches prometteuses en matière d'appel d'offres. Tout d'abord, la SNCF recourt de plus en plus aux variantes dans ses appels d'offres. Ces initiatives mettent en évidence un changement de routines en matière d'achat au sein de la SNCF. Il s'agit d'un «changement de culture » considérable pour l'entreprise publique (Entretien direction achat, 10/05/2016) qui est étroitement lié à la stratégie de ressources humaines de la SNCF. Le choix de donner plus de pouvoir de décision aux fournisseurs rencontre celui d'externaliser de plus en plus de fonctions auparavant exercées par les cheminots. Par conséquent, la SNCF réduit de fait son emprise sur la base de connaissances ferroviaires: elle maîtrise de moins en moins de connaissances du système ferroviaire et dépend davantage de ses fournisseurs pour certaines compétences. 
Par ailleurs, la SNCF propose maintenant des partenariats d'innovation, possibles depuis la transposition d'une directive européenne en droit français en avril 2016. Les partenariats d'innovation permettent de rassembler dans un même marché l'acquisition de prestation de recherche et développement et de biens et services issus de cette R\&D. C'est l'outil juridique qui a été choisi pour lancer le chantier de "TGV du futur»entre la SNCF et Alstom ${ }^{4}$, ce qui permet également la collaboration de l'opérateur dans le processus de production du matériel. Ce partenariat s'inscrit dans le cadre de la stratégie du gouvernement «nouvelle France industrielle » ayant conduit à la fondation d'une co-entreprise, «Speedinnov», entre l'ADEME (l'agence nationale de l'environnement) et Alstom. L'implication de l'Etat rappelle la logique du grand programme étatique historiquement à l'œuvre pour le projet TGV mais selon un régime qui donne un rôle plus important à Alstom que lors de la conception du TGV (Speck, 2003).

A travers ces deux exemples, le régime des appels d'offres s'affirme comme l'un des principaux leviers d'innovation ferroviaire en tant que mode de coordination des acteurs dans le cadre d'un régime marchand. Le partenariat d'innovation nuance quant à lui quelque peu les logiques de marché en offrant un cadre aux échanges entre opérateurs et constructeurs.

\subsubsection{La production des normes et des règles ferroviaires}

Les exploitants acquièrent des matériels ayant été préalablement autorisés par les agences nationales de sécurité ferroviaire, l'EPSF en ce qui concerne la France, qui examinent leur conformité aux règles européennes et nationales. Alors que les opérateurs historiques étaient de fait en charge de cet examen, compris dans l'appel d'offre, l'ouverture des marchés de l'industrie ferroviaire a nécessité la mise en place d'une autorité tierce en charge de cette tâche. L'étape de l'autorisation est ainsi devenue la dernière étape d'une procédure d'appel d'offre, après que la conformité de la proposition du constructeur au cahier des charges a été établie et au moment où la production débute. C'est au constructeur d'obtenir cette autorisation. Cette étape détermine en partie le «time to market » c'est-àdire la capacité à vendre plus rapidement le matériel conçu et produit. Ce «time to market» est identifié par la filière ferroviaire comme un enjeu particulièrement crucial pour sa performance et sa capacité à innover (Commission européenne, 2011).

D'un point de vue réglementaire, le cadrage porte principalement sur la sécurité et sur l'interopérabilité européenne (Fontanel et Christeller, 2016). Deux principaux corpus juridiques s'appliquent. Les trains européens doivent dorénavant se conformer aux STI (Spécifications Techniques d'Interopérabilité) qui sont des règlements européens. L'objectif de la politique d'interopérabilité européenne est non seulement de rendre possibles les circulations transfrontalières mais bien plus largement d'ouvrir les marchés nationaux en rendant obligatoire la compatibilité des systèmes techniques et en unifiant les règles de sécurité à l'échelle européenne. S'applique également une règlementation nationale, «document de référence national» (DRN) pour le cas de la France. Celui-ci transpose les directives européennes concernant la sécurité ferroviaire, qui s'articulent ellesmêmes étroitement aux STI. Si l'objectif des règles européennes est de parvenir à une règlementation commune en matière de sécurité du système ferroviaire européen, les règlementations nationales ajoutent des éléments règlementaires supplémentaires renvoyant aux spécificités de leur réseau (alimentation électrique par exemple) $^{5}$. Sur la base de ces corpus, les agences nationales de sécurité délivrent une autorisation de mise en exploitation commerciale (AMEC).

Par ailleurs, le secteur ferroviaire s'est entendu à l'échelle européenne pour standardiser le matériel ferroviaire, au-delà de la seule question de la sécurité. Il s'agit des «normes européennes » qui sont donc des normes privées définies directement par des représentants du secteur, opérateurs et, dans une

\footnotetext{
${ }^{4}$ Le partenariat d'innovation a été signé le 30/08/2016.

${ }^{5} \mathrm{D}$ 'après nos entretiens, environ $90 \%$ des règles sont harmonisées à l'échelle européenne. Les spécificités nationales sont donc de plus en plus limitées.
} 
moindre mesure, constructeurs, au sein du comité européen de normalisation. Ce corpus privé est toutefois étroitement lié au corpus juridique. En effet, les normes doivent être conformes aux règles européennes. Plus encore, les premières versions des STI ont eu tendance à recopier les normes. En un certain sens, les normes ont alors eu force de loi. Aujourd'hui, la «nouvelle approche» consiste à ce que les STI continuent de s'appuyer sur les normes, qui ont donc pour fonction de les appliquer aux différents cas et de les préciser, mais sans les répéter. Dans l'autre sens, les normes vont progressivement valoir présomption de conformité à la règle (Fontanel et Christeller, 2016). Derrière cette articulation étroite entre normes et règles européennes, apparaît la forte dimension technique et le poids des acteurs du secteur dans la règlementation européenne et, inversement, la dimension politique de la normalisation européenne, puisque cette dernière a un réel impact juridique.

En ce sens, le corpus de règles et de normes européennes ne doit pas être analysé comme une contrainte extérieure pour le secteur ferroviaire. Au contraire, il témoigne de la capacité du secteur ferroviaire à produire non seulement les normes mais plus encore les règles qui l'encadrent. D'un côté, les normes sont le fruit du consensus du secteur, au sein duquel les acteurs dominants sont les opérateurs historiques. De l'autre côté, les STI sont largement écrites dans le cadre de groupes de travail animés par l'agence ferroviaire européenne (ERA), qui est force de proposition pour mettre un sujet à l'agenda (puis portées par les directions de la commission européenne).

Les processus de production de ces textes correspondent donc à des jeux d'acteurs un peu différents. En particulier, les industriels ont plus de poids et d'influence au sein du processus de production des STI, l'ERA pouvant relayer directement leurs demandes, qu'au sein de celui des normes, fonctionnant sur le consensus aux échelles nationale et européenne. Le poids des exploitants dans le processus de normalisation peut être analysé comme un frein pour l'innovation ferroviaire, dans la mesure où les opérateurs tendent à être des garants très pointilleux du niveau de sécurité et des procédures qui la garantissent historiquement. Toutefois, la prise en compte du processus de production des STI, qui confère une plus grande place aux industriels, nuance ce jugement, d'autant plus que l'articulation entre les STI et les normes devient de plus en plus précise.

Finalement, à cette aune, le cadre normatif et règlementaire du ferroviaire apparait tout autant comme un potentiel levier d'innovation. Par exemple, à l'initiative de l'UNIFE, le secteur ferroviaire a mené plusieurs projets de recherche collaboratifs afin d'ajuster le cadre normatif et règlementaire pour rendre possibles certaines innovations, comme la virtualisation des essais (voir 2.1.3). La réussite de ces projets, qui ont conduit à une réécriture de certains textes normatifs, met en exergue la capacité du secteur ferroviaire à collaborer sur ces questions, c'est-à-dire à créer le consensus en faveur de certaines innovations. En ce sens, ce travail collaboratif sur les normes et les règles peut être appréhendé comme une capacité collective à l'innovation organisationnelle. Cependant, cette innovation organisationnelle, collaborative par définition, est également très incrémentale par nature.

L'enjeu du «time to market», jugé trop long par la plupart des acteurs de la filière ferroviaire, renvoie donc moins aux contraintes règlementaires et normatives, dont le secteur maîtrise largement l'évolution, qu'aux processus parallèles de l'autorisation et de l'homologation (au sens des négociations clients-fournisseurs dans le cadre des appels d'offres). Un point dur de ces processus correspond aux phases d'essais qu'ils exigent.

\subsubsection{Les essais au cœur du « time to market »}

Les essais de nouveaux matériels ferroviaires en conditions réelles sur le réseau constituent une étape incontournable du processus de conception et d'innovation. Les STI et les normes européennes définissent pour chaque critère couvert le nombre et la nature des essais à réaliser. De plus, les cahiers des charges des opérateurs peuvent également exiger des essais pour examiner des aspects qui ne relèvent ni des normes, ni des règles. Les essais exigés lors du processus d'autorisation sont présentés comme les plus problématiques pour le « time to market» en termes d'immobilisation de capital : 
« Le problème n'est pas le temps mais la valeur immobilisée pendant ce temps-là. Or, le temps nécessaire à décrocher un contrat [correspond à] un nombre limité de personnes à travailler. Idem, les essais de validation interne à un constructeur, c'est une validation de solutions, ça se fait sur des sous-ensembles en cours de construction. (...) Le problème des essais d'autorisation, c'est que ça retarde la mise en exploitation de flottes entières de trains construits. » (Entretien UNIFE, 2016)

Cette étape s'avère particulièrement complexe et onéreuse : les contraintes du système ferroviaire sont multiples et fortes (techniques, organisationnelles, commerciales, légales, de sécurité, etc.). Les essais en réel coûtent cher, et se heurtent à la rareté des sites réels disponibles. En effet, la séparation entre les opérateurs et les gestionnaires d'infrastructure, principe au cœur des différents "paquets ferroviaires 》 européens, rend visibles les coûts d'occupation des voies pour des besoins autres que l'exploitation et, donc, nécessite de répercuter ces coûts sur les commanditaires des essais. Pour ces derniers, opérateurs ferroviaires le plus souvent, les essais seront pour eux beaucoup plus chers qu'au temps des entreprises intégrées, sans compter que les sillons sont précieux pour l'exploitation, et qu'ils seront d'autant moins nombreux et disponibles pour les essais de mise au point. Une solution peut être d'effectuer les essais auprès d'autres gestionnaires. C'est ce que propose Eurotunnel depuis 2015.

Face à ces difficultés, la principale solution usitée est de procéder à une partie des tests du matériel sur des centres d'essais. Aujourd'hui, Alstom va souvent à Velim en Tchéquie pour faire les essais de ses matériels « grande vitesse ». Si ceci peut être envisagé pour les plus gros industriels, le coût d'accès aux centres d'essais peut apparaître rédhibitoire pour les opérateurs plus petits, réalisant des produits techniques en petite série.

Le principal levier avancé est celui de recourir davantage à la simulation numérique en lieu et place de certains essais sur voies et sur banc. La conception de modèles de simulation a suscité récemment de nombreux projets de recherche collaboratifs entre opérateurs, constructeurs et universitaires, afin notamment de proposer des processus d'autorisation reposant sur la simulation virtuelle à inscrire dans les normes et les règles européennes (Hanley et al, 2014). Une partie des résultats de ces projets a d'ores et déjà été introduite dans les normes 2015. Ces dernières permettent donc de simuler certains tests lorsqu'il s'agit d'un train légèrement modifié ou d'exploiter un train déjà autorisé sur un nouveau réseau (Funfschilling et al, 2014, p. 9).

Finalement, les solutions qui s'esquissent face au problème des essais ont en commun d'associer simulation numérique, bancs d'essais et essais sur voies. Les bancs d'essais engendrent non seulement des coûts de construction, mais également de fonctionnement qui posent la question de leur modèle économique. Par ailleurs cette solution, qui ne pourra jamais simuler la diversité des environnements, ne sera qu'un premier pas, de même que la simulation numérique. L'expérimentation en milieu réel restera toujours incontournable. Enfin, l'approfondissement des techniques de simulations nécessite de partager des données entre gestionnaires d'infrastructure, opérateurs et acteurs académiques, ce qui achoppe pour l'instant sur des questions de propriété intellectuelle.

\subsubsection{La propriété intellectuelle et l'incitation à l'innovation}

Dans un contexte de coordination marchande, la propriété intellectuelle devient un enjeu crucial. Dès lors que les exploitants se retirent de la conception du matériel et commandent des matériels munis de toutes leurs bases fonctionnelles, c'est aux constructeurs et à leurs sous-traitants d'innover. Or, «les entreprises, quelle que soit leur taille respective, ne sont incitées à miser sur l'innovation que si elles peuvent s'approprier pendant un temps suffisant les bénéfices attendus de tels efforts 》 (Crampes et Encaoua, 2003 in Lallement, 2009). Le partage des revenus issus de l'innovation est donc la question clef sous-jacente aux négociations tenant à la propriété intellectuelle.

Cet enjeu est particulièrement crucial pour les PME. Dans les réseaux impliquant divers partenaires de différentes tailles (clients, fournisseurs, organismes de recherche, centres de formation, etc.), les 
grands groupes du ferroviaire, comme ceux de l'automobile, laissent souvent l'innovation aux PME : on attend ainsi des sous-traitants de livrer des fonctions entières. Or, ces PME n'ont pas la capacité financière, ni humaine, de mener des programmes de R\&D. Un rapport d'enquête de l'assemblée nationale de 2011 montre ainsi que l'une des rares ETI implantée en France, GHH Valdunes, qui compte 900 salariés et 200 millions d'euros de chiffre d'affaires, n'a par exemple les moyens d'employer que deux salariés en $\mathrm{R} \& \mathrm{D}^{6}$.

Dans ce contexte, le régime de partage de la propriété intellectuelle entre entreprises au sein de la filière ferroviaire est décisif. Il détermine une partie cruciale des revenus des PME et donc leur motivation et leur capacité à innover. Les droits de propriété intellectuelle (DPI) sont conçus pour permettre aux PME de réguler leurs relations avec des acteurs plus puissants car de plus grande taille. Les brevets ou les licences d'utilisation accordent des quasi droits de propriété sur les connaissances, et permet à tout type d'acteurs d'en tirer des revenus. Mais les négociations d'exploitation commerciale des innovations se heurtent au déséquilibre des rapports de force au sein des chaines d'innovation.

Deux problèmes apparaissent sur ce sujet : un problème de ressources financières et humaines, et un problème de flexibilité des partenariats. Le problème des ressources recouvre à la fois le coût des brevets et leur complexité d'obtention. "Ceci implique qu'innover en partenariat avec de grandes firmes les expose à des risques souvent dissuasifs, en réduisant leurs capacités à s'approprier les résultats de telles activités de co-innovation » (OCDE, 2008 in Lallement, 2009). Les PME préfèrent donc aux brevets les licences, qui leur assurent un loyer contre l'exploitation des inventions, et qui sont plus adaptées à leurs produits ou prestations. Les DPI sont mobilisés par les grands groupes comme moyen d'exclure les autres de l'utilisation des idées et des inventions d'une entreprise en tentant de bloquer leurs concurrents par ce biais ou encore en laissant ces brevets « dormir » (Entretien Alstom, 2015). Ensuite, l'application stricte du modèle des DPI nuit à l'élargissement ou au renouvellement des partenariats, c'est-à-dire à leur flexibilité. Bâtir de nouveaux partenariats devient dès lors complexe, reposant en amont sur la signature de clause de confidentialité avant même le projet.

Repenser la mise en œuvre des DPI peut donc constituer un levier d'innovation pour la filière ferroviaire. Leur garantie en matière d'appel d'offre est une condition de l'innovation des fournisseurs. La définition de nouveaux modèles de PI est d'ailleurs mise à l'agenda de la filière dans le cadre de l'écriture d'une charte de l'innovation. Enfin, mettre en œuvre des partenariats avec des entreprises d'autres secteurs peut permettre de clarifier la question des droits d'exploitation commerciale, les clauses d'exclusivité étant définies sur un domaine d'exploitation tel que le ferroviaire. Ainsi, chez Alstom Transport, ce sont plus de 80 nouveaux partenariats innovants qui sont signés chaque année, principalement avec des PME et quelques grands groupes ne travaillant pas, majoritairement, dans le secteur du transport ferroviaire (automobile, aéronautique). Si des modèles de charte de PI peuvent constituer une ébauche de solution, celle-ci repose avant tout sur l'animation de la filière.

\subsection{L'évolution de l'écosystème ferroviaire et l'animation de la filière}

Au-delà du processus de production des matériels ferroviaires, les mutations du SSIP ferroviaire bouleversent également l'écosystème ferroviaire, en termes de modalités de la formation des cadres (2.2.1), de l'arrivée de nouveaux entrants (2.2.2) et concernant les ressources et les politiques de soutien à l'innovation (2.2.3).

\subsubsection{Les modalités de la formation et du recrutement des cadres et ingénieurs dans le ferroviaire}

Si un certain nombre d'innovations passées dans le ferroviaire peut être expliqué, au moins en partie, par l'excellence des filières de formation des ingénieurs en France, l'existence d'une filière de formation des ingénieurs dans le ferroviaire est aujourd'hui remise en cause, comme en témoigne la

\footnotetext{
${ }^{6}$ Cependant, Valdunes a été racheté en 2014 par MA Steel, leader chinois de l'industrie sidérurgique, qui a depuis investi dans un centre de R\&D au sein de Valdunes, renommé MG Valdunes. Les moyens sont donc vraisemblablement plus importants maintenant.
} 
baisse des étudiants dans les formations ferroviaires (Ingénieurs et Scientifiques de France, 2014 ; Observatoire de la Métallurgie, 2015).

Cette fragilisation de la filière de formation des ingénieurs dans le ferroviaire peut poser un problème de pérennité de la base de connaissances et de capacité de renouvellement du régime technologique ferroviaire (manque de compétences pour l'innovation dans de nouvelles thématiques). Une étude sur les formations concernant les industries ferroviaires (Observatoire de la Métallurgie, 2015) met en évidence une carence dans la part d'enseignement consacrée à la gestion de projet (et notamment de projet d'innovation), la faiblesse des formations sur les enjeux du «Design to Cost » et de la « Maintenabilité » (approches en coût global de possession), comme le montre l'absence d'offre de formation d'ingénieur en maintenance prédictive, et enfin concernant la maîtrise de la robotique.

Plus encore, les pratiques de formation des cadres par compagnonnage auprès de leurs aînés seraient un facteur de conformisme, renforçant la culture des «spécificités techniques 》 du ferroviaire, contraire à l'esprit d'innovation, en tout cas à l'innovation radicale. En effet, si ce système garantit une continuité des savoir-faire, et éventuellement une dynamique d'innovations incrémentales, il est avant tout fondé sur le principe du retour d'expérience, pouvant entraver la perspective d'innovations de rupture. Il en résulte une grande homogénéité des cadres du ferroviaire en termes de formation mais aussi d'imaginaire et de visions, qui renvoie aussi plus largement aux politiques de recrutement. Plus encore, ces deux aspects sont liés. Une formation par compagnonnage est nécessaire lorsque l'offre de formations est absente ou inadaptée à de nombreux domaines du ferroviaire.

Toutefois, la filière, à travers l'action des institutions d'animation du ferroviaire et des organismes de soutien à l'innovation, a bien identifié cet enjeu comme un potentiel levier d'innovation. L'initiative de Fer de France ${ }^{7}$ de mettre en place un cycle interprofessionnel, intitulé "Moisson-Desroches", pourrait être un élément allant à l'encontre de ce cercle vicieux. Ce cycle de formation des cadres souhaite transmettre une vision transversale de la filière tenant compte des mutations de l'écosystème ferroviaire.

\subsection{2. « Nouveaux entrants » dans l'écosystème ferroviaire », leviers de l'innovation ?}

Si l'organisation de la filière ferroviaire est bouleversée par le moindre leadership exercé par la SNCF, elle connait également un renouvellement important avec l'arrivée de nouveaux entrants. Ces derniers, majoritairement situés en aval de la filière (production des services ferroviaires), sont très divers : nouveaux opérateurs ferroviaires bien sûr, suite à la libéralisation du fret ferroviaire, mais surtout nouveaux concessionnaires de certaines lignes (Eurotunnel, LGV, Ports), issus du renouvellement de la gouvernance des infrastructures de transport, affirmation des collectivités locales (intercommunalités, régions) en tant qu'autorités organisatrices des transports suite à la décentralisation des services urbains et régionaux, initiatives d'entreprises d'autres secteurs s'intéressant à la mobilité, telles que les acteurs des TIC, et enfin l'implication de plus en plus nette des usagers et plus largement d'acteurs de la société civile, notamment via les outils numériques.

Ces nouveaux entrants sont porteurs de pratiques et d'approches différentes des activités ferroviaires, et en ce sens peuvent être des leviers d'innovation à divers titres. Ainsi la décentralisation des politiques de transport vers les collectivités locales a-t-elle conduit à prioriser les innovations sur le confort, le bruit et l'information voyageurs, principales demandes des usagers et des riverains. Parallèlement, les nouveaux concessionnaires se sont affirmés comme des acteurs apportant des innovations, notamment organisationnelles, en matière d'infrastructure en termes de gestion des voies, de maintenance: voie sur grave bitume (TGV Est), organisation des travaux, modernisation des équipements de l'infrastructure, système d'alimentation électrique par exemple. Enfin, les nouveaux

\footnotetext{
${ }^{7}$ Fédération réunissant les acteurs français du ferroviaire depuis les autorités organisatrices jusqu'aux industriels en passant par les opérateurs.
} 
services (numériques) aux voyageurs ont été majoritairement introduits par des acteurs du secteur des TIC, avant d'être parfois repris par les opérateurs ou les autorités organisatrices des transports.

Par ailleurs, certaines initiatives de la société civile peuvent aussi être analysées comme des leviers d'innovation. Il s'agit tout d'abord des opportunités offertes par la transition numérique, et notamment la multiplication des échanges d'informations entre voyageurs via les réseaux sociaux ou des plateformes et sites dédiés, qui répondent à un véritable besoin. Finalement, ces innovations numériques, qu'elles viennent des usagers ou directement des acteurs des TIC, ont été un élément déclencheur de l'innovation numérique par les opérateurs et les autorités organisatrices de transport: applications à destination des voyageurs, équipement des agents en divers outils numériques pour l'exploitation des services et les relations avec les passagers. Le régime technologique ferroviaire a ainsi connu un véritable renouvellement grâce à l'action de ces nouveaux entrants dans toute leur diversité. Ensuite, l'implication de la société civile prend également la forme d'une demande sociale de plus en plus pressante sur le sujet de la réduction des nuisances, souvent relayée par les collectivités locales aujourd'hui en charge des services de transports. Cette demande est par exemple à l'origine de projets de recherche pour diminuer le bruit des freinages.

Ces différents éléments sur le rôle des usagers et de la société civile montrent que des innovations peuvent avoir lieu très en aval au sein du cycle de production des services ferroviaires. Afin de faire de ces initiatives un véritable levier d'innovation, il convient de définir des interfaces à même relayer ces initiatives. Par exemple, la convention TER en Picardie entre le conseil régional et la SNCF a été l'occasion de mettre en place un «MiniLab» pour susciter des innovations locales avec les usagers eux-mêmes.

\subsubsection{Les ressources et les politiques de soutien à l'innovation ferroviaire}

L'analyse des processus des innovations récentes dans le secteur ferroviaire met en évidence un fonctionnement très cloisonné : les projets d'innovation sont très segmentés et généralement menés entreprise par entreprise. Ce cloisonnement est en partie lié à l'évolution des nouveaux modes d'organisation et de financement de la recherche par appels à projets, via l'Agence Nationale de la Recherche mais aussi le PREDIT (programme de recherche dans les transports) ou l'ADEME. Ce cloisonnement découle aussi des difficultés d'accès aux ressources stratégiques pour l'innovation ferroviaire, voire de l'inadéquation des institutions porteuses de ces ressources. En effet, de nombreuses ressources potentiellement stratégiques, qu'il conviendrait d'articuler dans les projets d'innovation ferroviaire, sont partagées entre plusieurs Instituts de Recherche Technologique, Railenium bien sûr, mais aussi Jules Verne (matériaux), SystemX (numérique), et plusieurs pôles de compétitivité, I-Trans (transport) mais aussi Nov@log (logistique) et Moveo (mobilité) ainsi que Systematic (numérique).

De plus, le fonctionnement de ces derniers soulève aussi un problème d'échelle géographique des pôles de compétitivité pour le ferroviaire. Le pôle référent pour le ferroviaire, I-Trans, est localisé dans le Nord-Pas-de-Calais et cultive son ancrage régional. Or, la géographie des centres de recherche et d'innovations ferroviaires, à la différence de celle des centres de production, n'est pas particulièrement articulée autour de cette région. Plus fondamentalement, le système d'innovation ferroviaire se déploie avant tout aux échelles nationale et européenne et s'avère peu régionalisé. En cela, le principe de pôles de compétitivité ancrés régionalement (c'est aussi le cas de Nov@log et de Moveo) pose des difficultés au secteur ferroviaire.

Enfin, ce cloisonnement renvoie également à l'insuffisance des relations entre les ingénieurs des opérateurs et des constructeurs et les chercheurs et universitaires.

Toutefois, plusieurs leviers sont d'ores et déjà disponibles pour renouveler les modalités de l'innovation dans le ferroviaire. Tout d'abord, les acteurs de la filière, opérateurs et industriels, ainsi que les chercheurs et universitaires, partagent les mêmes paradigmes techniques, preuve d'une grande 
proximité cognitive caractérisant le régime technologique et d'apprentissage ferroviaire. Notamment, apparait un relatif consensus entre industriels, opérateurs et organismes de recherche et d'innovation en faveur de plusieurs mutations du régime technologique ferroviaire : appliquer la transition numérique au ferroviaire, notamment en termes de normalisation et d'ingénierie (simulations numériques, voir 2.1.3), privilégier les innovations incrémentales dans le matériel roulant et les infrastructures, promouvoir des innovations organisationnelles pour la gestion du réseau et mettre l'accent sur l'offre de services vis-à-vis des voyageurs. Cette base de connaissances et ce diagnostic partagé des enjeux devraient grandement faciliter le dialogue au sein de la filière et rendre possible des projets d'innovation collaboratifs.

En effet, des initiatives collaboratives se multiplient petit à petit au sein du secteur, voire avec d'autres secteurs. Il s'agit par exemple de comités regroupant industriels et constructeurs pour juger les projets d'innovation de leurs fournisseurs mais aussi de «speed meetings » avec des start-ups, et de «hackathlon» (au sein de la SNCF et d'Alstom) permettant de tisser des liens avec les acteurs du numérique. Les initiatives d'animation de la filière par Fer de France à travers des « rail datings » ou à travers des projets collaboratifs ciblés, tel qu'Eurodigirail (mise en place de plates-formes collaboratives de dématérialisation des échanges au sein de la filière) s'inscrivent dans cette dynamique.

\section{Conclusion : quelles perspectives de recherche sur l'innovation ferroviaire?}

La grille d'analyse des SSIP nous a permis d'analyser les mutations et les contradictions du secteur ferroviaire en tenant compte du processus de production dans son ensemble et des multiples acteurs qui l'animent.

Il ressort de cette analyse que l'organisation de la filière ferroviaire a été particulièrement bouleversée par la montée en puissance des règles de concurrence à l'échelle européenne, c'est-à-dire par le déploiement progressif d'un régime marchand au sein de la filière ferroviaire. Le rôle et la position des opérateurs publics historiques en tant que leaders de la filière ferroviaire depuis la conception des matériels jusqu'aux services de transport ont été par conséquent considérablement remis en question. Or, le moindre leadership des opérateurs publics historiques soulève des enjeux cruciaux de coordination et d'échanges au sein de la filière alors que, comme le souligne la littérature sur les SI, ces interactions sont indispensables à la dynamique d'innovations. En effet, l'intensification de la concurrence n'a pas encore été accompagnée par la mise en œuvre de modalités ou d'outils permettant cette coordination.

Par conséquent, la montée en puissance d'un régime marchand au sein du secteur ferroviaire se traduit plutôt aujourd'hui par différents freins à l'innovation que nous avons essayés de mettre en évidence au travers des nœuds du SSIP ferroviaire. Il apparait de manière transversale que les échanges sont particulièrement difficiles non seulement entre industriels, fortement en concurrence, mais aussi entre les opérateurs donneurs d'ordre et leurs constructeurs fournisseurs, les échanges étant souvent perçus comme une entrave à la concurrence.

Cependant, s'esquissent de nouveaux modes de coordination que l'on peut saisir à travers plusieurs initiatives récentes. On assiste par exemple à la mise en place d'instances de collaboration et d'échanges au sein du secteur ferroviaire, telles que S2R à l'échelle européenne. De plus, émergent des réseaux intersectoriels à même de catalyser des innovations en matière d'applications numériques, de nouveaux matériaux ou encore de nouvelles organisations.

Finalement, cet article nous permet d'identifier plusieurs axes pour de futures recherches sur les innovations ferroviaires. 
Premièrement, à partir de l'approche en termes de SSIP, plusieurs travaux sont envisageables et souhaitables. Tout d'abord, il s'agirait de préciser la manière dont les mutations du Système National d'Innovation, à la fois français et européen (Amable et al, 1997; Boyer, 2015), ont joué sur les différents éléments du SSIP ferroviaire et sur ses nœuds alors que cet article ne les a que rapidement identifiés. Ensuite, il conviendrait d'effectuer une comparaison précise du SSIP ferroviaire par rapport aux SSIP habituellement étudiés par cette littérature (pharmacie, TIC, logiciels, chimie, machinesoutils, services : Malerba, 2004). Cette comparaison permettrait de mieux saisir dans quelle mesure les spécificités d'un secteur jouent sur les différents éléments de cette grille d'analyse, telles que le caractère hybride du ferroviaire, à la fois public et privé, ou encore la situation de monopsone où l'opérateur est le seul demandeur des constructeurs (dans la mesure où il est très difficile pour les industriels d'exporter leur production hors de leurs marchés d'origine). Enfin, il reste à analyser les effets réciproques des mutations du SSIP et des évolutions des routines des entreprises du ferroviaire et de leur manière de manager l'innovation.

Deuxièmement, un autre axe de recherche consisterait à effectuer une comparaison internationale des SSIP ferroviaires. Cette comparaison permettrait potentiellement d'analyser différents modes de coordination en vigueur dans le secteur et ainsi de mieux saisir dans quelle mesure ces interactions sont indispensables à la dynamique d'innovations, comme le souligne la littérature sur les SI. De cette façon, nous serions à même de distinguer les éventuelles coordinations nécessaires en raison de la technologie ferroviaire et celles qui découleraient plutôt de l'héritage du système précédent imprégné par le pilotage public. Cette perspective comparatiste serait particulièrement prometteuse à l'échelle européenne. En effet, tous les Etats-membres de l'Union Européenne sont de fait pris dans cette transition vers le régime marchand prévu par les règles européennes. Le cas de l'Allemagne semble incontournable. Le système ferroviaire allemand articule à la fois une domination historique de la Deutsche Bahn - il serait très intéressant d'analyser sa stratégie d'innovation au regard de celle de la SNCF -, l'existence d'un tandem solide entre le transporteur et l'industriel Siemens et une large ouverture à la concurrence des services régionaux (Guihéry, 2011; Schöller-Schwedes, 2010). Le cas de l'Italie est également très riche. Deux opérateurs, Trenitalia (opérateur historique public) et Nuovo Trasporto Viaggiatori (NTV), y sont en compétition sur la grande vitesse, cas unique en Europe, et son tissu industriel a connu des mutations considérables suite au rachat d'Ansaldo, le principal constructeur ferroviaire italien, par le japonais Hitachi, 15 ans après le rachat de Fiat-Ferroviaria par Alstom (Bergantino et al, 2015).

Troisièmement, il conviendrait d'approfondir l'analyse du poids de l'action publique dans les processus d'innovation ferroviaire selon plusieurs directions. Tout d'abord, afin de prolonger notre étude de la production des normes et des règles européennes, il s'agirait de réaliser une économie politique des relations à l'échelle européenne entre les acteurs du secteur ferroviaire, les institutions nationales et les institutions européennes. Ensuite, un travail ciblé sur les politiques de soutien à l'innovation ferroviaire permettrait d'en peser la cohérence par rapport à la manière dont fonctionne le ferroviaire, en particulier du point de vue des échelles géographiques (Torre, 2015). Enfin, l'analyse des interfaces de l'innovation situées en aval de la filière, fruits des initiatives de la société civile et des acteurs de la gouvernance locale des services de transport, constituerait un axe de recherche complémentaire à ce travail sur la production ferroviaire.

\section{Bibliographie}

[AMA 03] Amable B., Les systèmes d'innovation, dans P. MustaR, H. PenAN (dir.), Encyclopédie de l'innovation, Economica, Paris, p. 367-382, 2003.

[AMA 97] AMABle B., BARRE R. BOYER R., Les systèmes d'innovation à l'ère de la globalisation, Economica, Paris, 1997.

[BER 15] Bergantino A., CAPOzZa C. CAPurso M., "The impact of open access on intra - and inter - modal rail competition. A national level analysis in Italy", Transport Policy, n 39, p. 77-86, 2015. 
[BOY 15] BOYER R., Economie Politique des capitalismes, La découverte, Paris, 2015.

[BRE 97] BRESCHI S., MALERBA F., Sectoral Innovation Systems: Technological Regimes, Schumpeterian Dynamics, and Spatial Boundaries, in C. EDQUIST (ed.), Systems of Innovation. Technologies, Institutions and Organisations, Pinter Publishers, London, p. 130-156, 1997.

[BRE 00] Breschi S., MALERBA F., OrSENIGO L., "Technological Regimes and Schumpeterian Patterns of Innovation", Economic Journal, vol 110, n 463, p. 388-41, 2000.

[COM 11] COMMISSION EuROPÉENNE, Roadmap to a Single European Transport Area - Towards a competitive and resource efficient transport system, Livre Blanc, 2011.

[CRA 03] Crampes C., EnCaOua D., Microéconomie de l'innovation, dans P. Mustar, H. Penan (dir.), Encyclopédie de l'innovation, Economica, Paris, p. 405-430, 2003.

[CRO 01] CROZET Y., «Réforme et concurrence dans le transport ferroviaire européen, bilan et perspectives », Realites Industrielles-Annales des Mines, p. 27-35, février 2001.

[CRO 14] CROZET Y., 30 fiches pour comprendre les enjeux de la réforme ferroviaire, Avril 2014. URL: http://www.mobilettre.com/wp-content/uploads/2014/05/30-fiches-pour-comprendre-les-enjeux-de-la$\mathrm{r} \% \mathrm{C} 3 \% \mathrm{~A} 9$ forme-ferroviaire.pdf

[CRO 11] CROzet, Y., Desmaris, C., «Le transport ferroviaire régional de voyageurs : un processus collectif d'apprentissage », Recherche Transports Sécurité (RTS), vol 27, n 3, p. 143-162, 2011.

[FON 16] FONTANEL E., CHRISTELLER R., Le matériel roulant dans le système ferroviaire - Tome 1, La vie du rail, Paris, 2016.

[FOU 03] FOURNIAU J-M., Introduction, Revue d'histoire des chemins de fer, $\mathrm{n}^{\circ} 26,2003$. URL : http://rhcf.revues.org/1924

[FUN 14] FUNFSCHILling C., BEZIN Y., SEBÈS M., "DynoTRAIN, introduction of simulation in the certification process of railway vehicles", Transport Research Arena, April 14-17 2014, Paris.

[GUI 11] GUIHÉRY L., " Transport ferroviaire régional en Allemagne : 1'exemple de la région de Leipzig », Recherche Transport Sécurité, vol 27, n 106, p. 163-177, 2011.

[HAN 14] Hanley R., Licciardello R., Martin C., Rose R. (2014), "TriotRAin: bringing research and standardisation closer together", Transport Research Arena, April 14-17 2014, Paris.

[ING 14] INGÉNIEURS ET SCIENTIFIQUES DE FRANCE, La filière ferroviaire française à la croisée des chemins. Comprendre la situation et les enjeux, Cahier n¹5, Comité Transports, 2014.

[LAL 09] LALLEMENT R. (2009), « Propriété intellectuelle, innovation et développement des PME en France », revue de l'IRES, $\mathrm{n}^{\circ}$ 3, p. 159-189, 2009.

[LEG 95] LE GALÈS P., « Du gouvernement des villes à la gouvernance urbaine », Revue française de science politique, vol. $45, \mathrm{n}^{\circ} 1,57-95,1995$.

[MAC 03] MACHEFERT-TASSIN Y, «La Commission électrotechnique internationale (CEI) et le Comité français (CEF). Normes et recommandations, 1948-1988 », Revue d'histoire des chemins de fer, $\mathrm{n}^{\circ} 26,2003$. URL: http://rhcf.revues.org/1956

[MAL 02] MALERBA F, «Les régimes technologiques et les systèmes sectoriels d'innovation en Europe », dans J-P. TOUFfuT (dir.), Institutions et innovations. De la recherche aux systèmes sociaux d'innovation, Albin Michel, Paris, $\mathrm{p}$. 232-247, 2002.

[MAL 04] MALERBA F., Sectoral Innovation Systems in Europe, Cambridge University Press, Cambridge, 2004.

[MIN 16] Ministère de L'EnViRonnement, DE L'Energie et DE la Mer, Comptes des Transports 2015, 53 rapport de la Commission des comptes des transports de la Nation, Paris, 2016.

[NAS 08] NASH C., "Passenger railway reform in the last 20 years - European experience reconsidered", Research in Transportation Economics, ${ }^{\circ}$ 22, p. 61-70, 2008.

[NAS 10] NASH C., "European rail reform and passenger services e the next steps", Research in Transportation Economics, n 29, p. 204-211, 2010.

[OBS 15] OBSERVATOIRE DE LA MÉTALLURGIE, Etude prospective sur la filière matériel roulant ferroviaire horizon 2015 -2025, Rapport, 2015.

[OCD 04] OCDE, Compendium statistique de la science et de la technologie, Rapport, 2004. 
[OCD 08] OCDE, Open Innovation in Global Networks, Rapport, 2008.

[PER 14] PÉRENNES P., Spécificité du secteur ferroviaire et libéralisation: la question du signal prix, Thèse de doctorat, Université Paris 1 Panthéon Sorbonne, Paris, 2014.

[RIB 03] RIBEILL G., « Diversité ou unité des exploitations ferroviaires : un antagonisme permanent ? », Revue d'histoire des chemins de fer, $\mathrm{n}^{\circ}$ 26, 2003. URL : http://rhcf.revues.org/1937

[SET 13] SETRA/CERTU, Les matériels ferroviaires de voyageurs sur le réseau ferré national, Rapport, 2013. URL : http://www.infra-transports-materiaux.cerema.fr/IMG/pdf/1344w-Rapport_materiel_roulant.pdf

[SCH 10] SCHÖLLER-SCHWEDES O., "The failure of integrated transport policy in Germany: a historical perspective", Journal of Transport Geography, vol. 18, n 1, p. 85-96, 2010.

[SPE 03] SPECK K., « Système national d'innovation et TGV - Ce qu'une grille de lecture de l'économie de l'innovation peut apporter à l'analyse des évolutions d'un système technique... et réciproquement », Recherche Transports Sécurité, $\mathrm{n}^{\circ}$ 78, p. 33-41, 2003.

[THE 05] THEYS J., Quelles technologies clefs pour l'Europe? Les enjeux liés au transport, Rapport pour la DG recherche de la Commission Européenne, 2005.

[TOR 15] TORRE A., «Théorie du développement territorial », Géographie, économie, société, vol 17, n 3, p. 273 $288,2015$. 